\title{
Identification of genome compositions in allopolyploid species of the genus Elymus (Poaceae: Triticeae) in the Asian part of Russia by CAPS analysis
}

\author{
A.V. Agafonov ${ }^{1} \otimes$, E.V. Shabanova (Kobozeva) ${ }^{1}$, S.V. Asbaganov ${ }^{1}$, A.V. Mglinets ${ }^{2}$, V.S. Bogdanova ${ }^{2}$ \\ ${ }^{1}$ Central Siberian Botanical Garden of Siberian Branch of the Russian Academy of Sciences, Russia, Novosibirsk, Russia \\ ${ }^{2}$ Institute of Cytology and Genetics of Siberian Branch of the Russian Academy of Sciences, Novosibirsk, Russia \\ 凶e-mail:agalex@mail.ru
}

\begin{abstract}
The genus Elymus L., together with wheat, rye, and barley, belongs to the tribe Triticeae. Apart from its economic value, this tribe is characterized by abundance of polyploid taxa formed in the course of remote hybridization. Single-copy nuclear genes are convenient markers for identification of source genomes incorporated into polyploids. In the present work, a CAPS-marker is developed to distinguish basic $\mathrm{St}, \mathrm{H}$, and $\mathrm{Y}$ genomes comprising polyploid genomes of Asiatic species of the genus Elymus. The test is based on electrophoretic analysis of restriction patterns of a PCR-amplified fragment of the gene coding for beta-amylase. There are about 50 Elymus species in Russia, and most of them are supposed to possess one of three haplome combinations, StH, StY and StHY. Boreal $\mathrm{StH}-\mathrm{genomic}$ species endemic for Russia are the least studied. On the basis of nucleotide sequences from public databases, Taql restrictase was selected, as it produced patterns of restriction fragments specific for St, $\mathrm{H}$, and $\mathrm{Y}$ haplomes easily recognizable in agarose gel. A sample of 68 accessions belonging to 32 species was analyzed. In 15 species, the earlier known genomic constitutions were confirmed, but in E. kamoji this assay failed to reveal the presence of $\mathrm{H}$ genome. This unusual $\mathrm{H}$ genome was suggested to originate from a different Hordeum species. In 16 species, genomic constitutions were identified for the first time. Fifteen accessions from Asian Russia possessed the genomic constitution StStHH, and E. amurensis, phylogenetically close to the StY-genomic species E. ciliaris, had the genomic constitution StStYY. It is inferred that the center of species diversity of the StH-genomic group is shifted to the north as compared to the center of origin of StY-genomic species, confined to China.
\end{abstract} Key words: Elymus; taxonomy; allopolyploids; genome constitution; CAPS markers.

For citation: Agafonov A.V., Shabanova (Kobozeva) E.V., Asbaganov S.V., Mglinets A.V., Bogdanova V.S. Identification of genome compositions in allopolyploid species of the genus Elymus (Poaceae: Triticeae) in the Asian part of Russia by CAPS analysis. Vavilovskii Zhurnal Genetiki i Selektsii =Vavilov Journal of Genetics and Breeding. 2020; 24(2):115-122. DOI 10.18699/VJ20.606

\section{Выявление геномного состава аллополиплоидных видов рода Elymus (Роaceae: Triticeae) Азиатской России с помощью CAPS-анализа}

\author{
А.В. Агафонов ${ }^{1} \otimes$, Е.В. Шабанова (Кобозева) $)^{1}$, С.В. Асбаганов ${ }^{1}$, А.В. Мглинец ${ }^{2}$, В.С. Богданова ${ }^{2}$ \\ ${ }^{1}$ Центральный сибирский ботанический сад Сибирского отделения Российской академии наук, Новосибирск, Россия \\ 2 Федеральный исследовательский центр Институт цитологии и генетики Сибирского отделения Российской академии наук, \\ Новосибирск, Россия \\ هe-mail:agalex@mail.ru
}

Аннотация. Род Elymus L. наряду с пшеницей, рожью и ячменем принадлежит к трибе Triticeae. Помимо своего хозяйственного значения, эта триба характеризуется широким распространением аллополиплоидных таксонов, которые формируются в ходе межвидовой и межродовой гибридизации и последующих преобразований вовлеченных в гибридизацию диплоидных геномов. Для идентификации исходных геномов в составе полиплоидов удобны малокопийные ядерные гены, менее подверженные процессам реорганизации, чем повторенные некодирующие элементы. В настоящей работе разработан удобный CAPS-маркер для различения базисных геномов St, H, Y, входящих в состав азиатских видов рода Elymus, с помощью электрофоретического анализа фрагментов рестрикции ПЦР-амплифицированного участка гена, кодирующего $\beta$-амилазу. В России распространено около 50 видов Elymus предположительно трех гапломных комбинаций: StH, StY и StHY. Наименее изученными остаются бореальные StH-геномные виды - эндемики Российской Федерации. По результатам анализа ранее изученных разными авторами нуклеотидных последовательностей гена $\beta$-амилазы была отобрана эндонуклеаза рестрикции Taql, которая имела различающиеся по положению сайты узнавания в составе вышеуказанного фрагмента из геномов St, H и Y. В результате расщепления ПЦРпродукта эндонуклеазой Taql у каждого из исходных гапломов формировался специфичный паттерн фраг- 


\begin{abstract}
ментов рестрикции, легко визуализируемый в агарозном геле. Проанализирована выборка из 68 образцов, принадлежащих 32 видам. У 15 видов была подтверждена ранее известная геномная конституция, у E. kaтоji этот метод не позволил выявить присутствие генома Н. Предполагается возможное происхождение данного варианта генома Н от другого вида Hordeum. У 16 видов геномная конституция определена впервые. Показано, что большинство изученных видов бореальной группы видов из Сибири и Российского Дальнего Востока имеют геномную конституцию StStHH. Исключение составил E. amurensis, филогенетически близкий к StY-геномному виду E. ciliaris и также имеющий геномный состав StStYY. Сделан вывод, что основное видовое разнообразие StH-геномной группы находится севернее центра происхождения большинства StY-геномных видов рода.
\end{abstract}

Ключевые слова: Elymus; таксономия; аллополиплоиды; геномная конституция; CAPS-маркеры.

\section{Introduction}

The genus Elymus L. is the largest in the tribe Triticeae Dum. and, according to different estimates, counts from 150 to 200 species (Dewey, 1984; Barkworth, 2000). It is represented only by alloploid taxa with genome compositions including several basic genomes (haplomes) in different combinations. The genetic basis of the genus Elymus is formed by five haplomes descending from different genera of the tribe Triticeae: (St) Pseudoroegneria, (H) Hordeum, (P) Agropyron, (W) Astralopyrum, (Y) donor unknown. Genome constitution was proposed as a stable genetic criterion for taxonomic classification of Elymus species (Löve, 1984). Within a relatively short span of time, substantial changes occurred in the taxonomy of the tribe Triticeae on the basis of the genomic system of classification suggested by D.R. Dewey (1984). During the next 20 years, six genera were identified according to variants of genome constitution: Douglasdeweya C. Yen, J.L. Yang \& B.R. Baum (PPStSt), Roegneria C. Koch (StStYY), Anthosachne Steudel (StStWWYY), Kengylia C. Yen \& J.L. Yang (PPStStYY), Campeiostachys Drobow (HHStStYY), and Elymus L. (StStHH, StStStHH, StStHHHH).

However, departing from A. Löve's principles, many botanists still attribute several genome combinations to the single genus Elymus s. 1. With all this, genome constitutions are not yet determined in about $40 \%$ of species (Okito et al., 2009). According to current evidence, 53 species of the genus Elymus subdivided into four sections occur in Russia (Tsvelyov, 2008; Tsvelyov, Probatova, 2010). Two of the sections, Elymus and Goulardia (Husn.) Tzvelev, contain species with different genomic constitutions, which obviously contradicts the phylogenetic principle of their formulation. We suppose that Russia is home to species with only three haplome combinations: StH, StY, and StHY (Agafonov et al., 2015). Boreal StH-genomic endemics of Russia are less studied. According to the taxonomic system based on the genome constitution, the Elymus species should be attributed to three genera: Elymus, Roegneria, and Campeiostachys. However, in our view, the division of the species inhabiting Russia into three genera is impractical due to the difficulties of morphologic identification of these genera. With all this, taxonomic classification within the genus based on genome constitutions is indispensable for the construction of a phylogenetically oriented taxonomy of the genus.

Earlier, Cleaved Amplified Polymorphic Sequences (CAPS) markers were used to distinguish individual genomes in representatives of the tribe Triticeae (Gostimsky et al., 2005; Li et al., 2007; Hu et al., 2014; Shavrukov, 2016). Some advantages of CAPS markers are their codominance, moderate sensitivity to the amount of genomic DNA, and relatively low cost.
We were first to use CAPS-markers to identify the genomic constitutions of species of the genus Elymus (Kobozeva et al., 2017). For this purpose, primers were designed based on the known sequences of the gene coding for $\beta$ amylase (MasonGamer, 2013), which included 38 sequences of haplome St, 23 of haplome $\mathrm{H}$, and 15 of haplome Y, belonging to 24 Elymus species. Of them, 14 species had the genomic composition StStHH; 9, StStYY; and 1, StStHHUkUk (Elytrigia repens). Variable positions were sought that would discriminate representatives of an individual genome from the other two. Special attention was paid to those genome-specific sequence variants that resulted in appearance/disappearance of recognition sites for restriction endonucleases. It was found that digestion of the PCR products with TaqI endonuclease resulted in the formation of genome-specific restriction patterns. In the present work, we apply CAPS analysis to a large sample of Elymus species from Asian Russia to reveal their genome constitutions unknown hitherto.

\section{Materials and methods}

Plant material included 68 accessions of the species with known (Table 1) and unknown (Table 2) genome constitutions found in Russia. The species nomenclature is given according to N.N. Tsvelyov and N.S. Probatova (2010). The accessions analyzed were received from the scientific collection of biological resources of the Central Siberian Botanic Garden SB RAS "Collections of living plants indoors and outdoors"; their identification numbers are given in Tables 1 and 2. Prefixes correspond to the geographic origin of the accessions.

Total DNA was extracted from $20 \mathrm{mg}$ of dried green matter with the use of NucleoSpin Plant II Kit (Macherey-Nagel, Germany) according to manufacturer's recommendations. Amplification of the $\beta$ amylase gene fragment was made in a C-1000 thermocycler (Bio-Rad, USA) with the following primers: El_balg_F4 (5'-GGTACCATCGTGGACATTGAA-3') and El_balg_R4 (5'-CTGTACCACCAGTGAATGCC-3') (Kobozeva et al., 2017). The PCR reaction mixture of $15 \mu \mathrm{L}$ in volume contained $1 \times$ buffer for Taq polymerase, $0.2 \mathrm{mM}$ each dNTP, $1.5 \mathrm{mM} \mathrm{MgCl}_{2}, 1 \mu \mathrm{M}$ each of primers, $20 \mathrm{ng}$ of genomic DNA, and $1 \mathrm{U}$ of HS Taq DNA polymerase (Eurogene, RF). The following settings were used: predenaturation at $94{ }^{\circ} \mathrm{C}$ for $4 \mathrm{~min} ; 40$ cycles: denaturation at $94{ }^{\circ} \mathrm{C}$ for $20 \mathrm{~s}$, primer annealing at $60{ }^{\circ} \mathrm{C}$ for $25 \mathrm{~s}$, elongation at $72{ }^{\circ} \mathrm{C}$ for $90 \mathrm{~s}$; postextension at $72{ }^{\circ} \mathrm{C}$ for 5 minutes. CAPS-analysis (Konieczny, Ausubel, 1993) was made as follows: $8 \mu \mathrm{L}$ of the $\mathrm{PCR}$ reaction mixture was mixed with $\mathrm{MQ}-\mathrm{H}_{2} \mathrm{O}$ and $\mathrm{Taq} \mathrm{I}$ buffer up to $1 \times$ concentration in a volume of $15 \mu \mathrm{L}$, and 1 unit of TaqI restrictase (Thermo Scientific, USA) was added. The mixture was incubated at $65{ }^{\circ} \mathrm{C}$ for 1 hour and resolved in 
Table 1. Accessions of Elymus species with known genomic constitutions determined by the classical cytogenetic method

\begin{tabular}{|c|c|c|c|}
\hline No & $\begin{array}{l}\text { Elymus species; } \\
\text { accession }\end{array}$ & $\begin{array}{l}\text { Genomic } \\
\text { constitutions }\end{array}$ & Location and collectors \\
\hline 1 & $\begin{array}{l}\text { E. kamoji; } \\
\text { CCH-1395 }\end{array}$ & $\begin{array}{l}\text { StHY } \\
\text { (Zhou et al., 1999) }\end{array}$ & $\begin{array}{l}\text { People's Republic of China (PRC), prov. Sichuan, Chengdu outskirts, a forest patch in Panda park, } \\
\text { alt. } 527 \mathrm{~m}, \mathrm{~N} 30^{\circ} 44.253^{\prime} \mathrm{E} 104^{\circ} 8.453^{\prime} \text { (A. Agafonov, E. Kobozeva) }\end{array}$ \\
\hline 2 & $\begin{array}{l}\text { E. kamoji; } \\
\text { SLA-1276 }\end{array}$ & $»$ & $\begin{array}{l}\text { Russia, Primorskiy Krai, Khasan raion, Slavyanka Town, coastal meadow, } \\
\text { alt. } 1 \mathrm{~m}, \mathrm{~N} 42^{\circ} 52.101^{\prime} \mathrm{E} 131^{\circ} 22.987^{\prime} \text { (A. Agafonov) }\end{array}$ \\
\hline 3 & $\begin{array}{l}\text { E. dahuricus; } \\
\text { BUD-0781 }\end{array}$ & $\begin{array}{l}\text { StHY } \\
\text { (Dewey, 1984) }\end{array}$ & $\begin{array}{l}\text { Russia, Republic of Buryatia, Dzhirga raion, Nizhniy Torey village outskirts, the Toreyka River } \\
\text { valley, meadow slope, alt. } 863 \mathrm{~m}, \mathrm{~N} 50^{\circ} 34.567^{\prime} \mathrm{E} 104^{\circ} 52.571^{\prime} \text { (N. Badmaeva) }\end{array}$ \\
\hline 4 & $\begin{array}{l}\text { E. dahuricus; } \\
\text { CHJ-1516 }\end{array}$ & $»$ & $\begin{array}{l}\text { People's Republic of China (PRC), Jilin Province, nearby Lake Tsagan-Nur, forest plantation, } \\
\text { alt. } 138 \mathrm{~m}, \mathrm{~N} 45^{\circ} 12.106^{\prime} \mathrm{E} 124^{\circ} 25.074^{\prime} \text { (S. Asbaganov) }\end{array}$ \\
\hline 5 & $\begin{array}{l}\text { E. schrenkianus; } \\
\text { AKA-0702 }\end{array}$ & $\begin{array}{l}\text { StHY } \\
\text { (Lu, Bothmer, 1992) }\end{array}$ & $\begin{array}{l}\text { Russia, Altai Republic, Kosh-Agach raion, stony meadow, Kalanegir River valley, } \\
\text { alt. } 2283 \mathrm{~m}, \mathrm{~N} 49^{\circ} 37.896^{\prime} \mathrm{E} 88^{\circ} 29.441^{\prime} \text { (S. Lukjanchikov) }\end{array}$ \\
\hline 48 & $\begin{array}{l}\text { E. schrenkianus; } \\
\text { AUK-0652 }\end{array}$ & 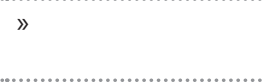 & $\begin{array}{l}\text { Russia, Altai Republic, Kosh-Agach raion, stony meadow in a brook valley, } \\
\text { alt. } 2445 \mathrm{~m}, \mathrm{~N} 49^{\circ} 30.418^{\prime} \mathrm{E} 88^{\circ} 5.012^{\prime} \text { (A. Agafonov, B. Salomon) }\end{array}$ \\
\hline 6 & $\begin{array}{l}\text { E. pendulinus; } \\
\text { VOK-0738 }\end{array}$ & $\begin{array}{l}\text { StY } \\
\text { (Jensen, 1990) }\end{array}$ & $\begin{array}{l}\text { Russia, Vladivostok City outskirts, Okeanskaya Station, shrubbery; } \\
\text { alt. } 7 \mathrm{~m}, \mathrm{~N} 43^{\circ} 14.10^{\prime} \mathrm{E} 132^{\circ} 0.19^{\prime} \text { (A. Agafonov) }\end{array}$ \\
\hline 7 & $\begin{array}{l}\text { E. pendulinus; } \\
\text { CHE-1044 }\end{array}$ & $»$ & $\begin{array}{l}\text { Russia, Altai Republic, Chemal raion, confluence of the Chemal and Katun Rivers, } \\
\text { alt. } 434 \mathrm{~m}, \mathrm{~N} 51^{\circ} 23.533^{\prime} \mathrm{E} 86^{\circ} 00.197^{\prime} \text { (E. Kobozeva) }\end{array}$ \\
\hline 8 & $\begin{array}{l}\text { E. gmelinii; } \\
\text { AUS-1013 }\end{array}$ & $\begin{array}{l}\text { StY (Jensen, Hatch, } \\
\text { 1989) }\end{array}$ & $\begin{array}{l}\text { Russia, Altai Republic, Shebalino raion, Ust-Sema settlement outskirts, shingle bank in the Katun } \\
\text { River left floodplain, alt. } 341 \mathrm{~m}, \mathrm{~N} 51^{\circ} 37.620^{\prime} \mathrm{E} 85^{\circ} 45.923^{\prime} \text { (A. Agafonov, E. Kobozeva) }\end{array}$ \\
\hline 9 & $\begin{array}{l}\text { E. sibiricus; } \\
\text { ACH-1601 }\end{array}$ & $\begin{array}{l}\text { StH } \\
\text { (Dewey, 1974) }\end{array}$ & $\begin{array}{l}\text { Russia, Altai Republic, Chike-Taman Pass, right side of the road, } 500 \mathrm{~m} \mathrm{~N} \text { of the viewpoint, } \\
\text { alt. } 1250 \mathrm{~m}, \mathrm{~N} 50^{\circ} 38.911^{\prime} \mathrm{E} 86^{\circ} 18.789^{\prime} \text { (E. Kobozeva) }\end{array}$ \\
\hline 10 & $\begin{array}{l}\text { E. caninus; } \\
\text { OSE-1423 }\end{array}$ & $\begin{array}{l}\text { StH } \\
\text { (Dewey, 1968) }\end{array}$ & $\begin{array}{l}\text { Russia, North Osetia-Alania Republic, Alagir raion, ecological path in the Tseydon River valley, } \\
\text { alt. } 1951 \mathrm{~m}, \mathrm{~N} 42^{\circ} 47.139^{\prime} \mathrm{E} 43^{\circ} 53.605^{\prime} \text { (A. Agafonov, M. Agafonova) }\end{array}$ \\
\hline 11 & $\begin{array}{l}\text { E. caninus; } \\
\text { OSE-1427 }\end{array}$ & $»$ & $\begin{array}{l}\text { Russia, North Osetia-Alania Republic, Iraf raion, Stur-Digora village outskirts, } \\
\text { Great Caucasus Mt. Range northern slope, the valley of a right Urukh River tributary, } \\
\text { alt. } 1996 \mathrm{~m}, \mathrm{~N} 42^{\circ} 52.898^{\prime} \mathrm{E} 43^{\circ} 35.959^{\prime} \text { (S. Asbaganov) }\end{array}$ \\
\hline 12 & $\begin{array}{l}\text { E. caninus; } \\
\text { UKU-1617 }\end{array}$ & $»$ & $\begin{array}{l}\text { Russia, Republic of Bashkortostan, Beloretskiy district, Novoabzakovo village outskirts, } \\
\text { dry meadow in a mixed forest, alt. } 618 \mathrm{~m}, \mathrm{~N} 53^{\circ} 48.718^{\prime} \mathrm{E} 58^{\circ} 40.377^{\prime} \text { (A. Agafonov, S. Asbaganov) }\end{array}$ \\
\hline 13 & $\begin{array}{l}\text { E. gmelinii; } \\
\text { BKA-0962 }\end{array}$ & $\begin{array}{l}\text { StY (Jensen, Hatch, } \\
1989)\end{array}$ & $\begin{array}{l}\text { Russia, Primorskiy Krai, Bolshoy Kamen Town outskirts, } \\
\text { alt. } 41 \mathrm{~m}, \mathrm{~N} 43^{\circ} 7.513^{\prime} \mathrm{E} 132^{\circ} 25.133^{\prime} \text { (A. Agafonov) }\end{array}$ \\
\hline 14 & $\begin{array}{l}\text { E. fedtschenkoi; } \\
\text { KSA-0935 }\end{array}$ & $\begin{array}{l}\text { StY } \\
\text { (Liu, Dewey, 1983) }\end{array}$ & $\begin{array}{l}\text { Kazakhstan, South Altai Mt. Range, the W principal slope, } \\
\text { alt. } 1791 \mathrm{~m}, \mathrm{~N} 49^{\circ} 05.077^{\prime} \mathrm{E} 86^{\circ} 04.483^{\prime} \text { (D. Gerus) }\end{array}$ \\
\hline 49 & $\begin{array}{l}\text { E. fedtschenkoi; } \\
\text { KME-1729 }\end{array}$ & $»$ & $\begin{array}{l}\text { Kazakhstan, Trans-lli Alatau Mts., Medeu village outskirts, stony highland meadow, } \\
\text { alt. } 3223 \mathrm{~m}, \mathrm{~N} 43^{\circ} 07.101^{\prime} \mathrm{E} 77^{\circ} 06.828^{\prime}(\mathrm{S} \text {. Asbaganov) }\end{array}$ \\
\hline 50 & $\begin{array}{l}\text { E. fedtschenkoi; } \\
\text { AUR-1714 }\end{array}$ & $»$ & $\begin{array}{l}\text { Russia, Altai Republic, Ulagan raion, the Yarly-Amry River, stony highland meadow, } \\
\text { alt. } 2180 \mathrm{~m}, \mathrm{~N} 50^{\circ} 19.044^{\prime} \mathrm{E} 087^{\circ} 43.049^{\prime} \text { (E. Kobozeva) }\end{array}$ \\
\hline 51 & $\begin{array}{l}\text { E. nevskii; } \\
\text { KME-1728 }\end{array}$ & $\begin{array}{l}\text { StY } \\
\text { (Dewey, 1980) }\end{array}$ & $\begin{array}{l}\text { Kazakhstan, Trans-lli Alatau Mts., Medeu village outskirts, stony highland meadow, } \\
\text { alt. } 3223 \mathrm{~m}, \mathrm{~N} 43^{\circ} 07.101^{\prime} \mathrm{E} 77^{\circ} 06.828^{\prime} \text { (S. Asbaganov) }\end{array}$ \\
\hline 15 & $\begin{array}{l}\text { E. fibrosus; } \\
\text { ABZ-1602 }\end{array}$ & $\begin{array}{l}\text { StH } \\
\text { (Dewey, 1984) }\end{array}$ & $\begin{array}{l}\text { Russia, Republic of Bashkortostan, Beloretsk raion, Novoabzakovo village outskirts, } \\
\text { a ground road side, alt. } 546 \mathrm{~m}, \mathrm{~N} 53^{\circ} 47.845^{\prime} \mathrm{E} 58^{\circ} 37.291^{\prime} \text { (A. Agafonov, S. Asbaganov) }\end{array}$ \\
\hline 16 & $\begin{array}{l}\text { E. mutabilis; } \\
\text { KHA-1210 }\end{array}$ & $»$ & $\begin{array}{l}\text { Russia, Krasnoyarskiy Krai, Shushenskoe raion, Shushenskiy Bor pine forest, } \\
\text { alt. } 495 \mathrm{~m}, \mathrm{~N} 52^{\circ} 49.622^{\prime} \mathrm{E} 91^{\circ} 26.609^{\prime} \text { (S. Asbaganov) }\end{array}$ \\
\hline 17 & $\begin{array}{l}\text { E. mutabilis; } \\
\text { ABZ-1607 }\end{array}$ & $»$ & $\begin{array}{l}\text { Russia, Republic of Bashkortostan, Beloretsk raion, Novoabzakovo village outskirts, } \\
\text { a ground road side, alt. } 546 \mathrm{~m}, \mathrm{~N} 53^{\circ} 47.845^{\prime} \text { E } 58^{\circ} 37.291^{\prime} \text { (A. Agafonov, S. Asbaganov) }\end{array}$ \\
\hline 26 & $\begin{array}{l}\text { E. abolinii; } \\
\text { BUD-0780 }\end{array}$ & $\begin{array}{l}\text { StY } \\
\text { (Jensen, 1989) }\end{array}$ & $\begin{array}{l}\text { Russia, Republic of Buryatia, Dzhida raion, Nizhniy Torey village outskirts, the Toreyka River } \\
\text { valley, meadowy slope, alt. } 863 \mathrm{~m}, \mathrm{~N} 50^{\circ} 34.634^{\prime} \mathrm{E} 104^{\circ} 52.781^{\prime} \text { (N. Badmaeva) }\end{array}$ \\
\hline 27 & $\begin{array}{l}\text { E. ciliaris; } \\
\text { VOK-0711 }\end{array}$ & $\begin{array}{l}\text { StY } \\
\text { (Dewey, 1984) }\end{array}$ & $\begin{array}{l}\text { Russia, Vladivostok Sity env., between Sanatornaya and Okeanskaya Stations, shrubbery, } \\
\text { alt. } 4 \mathrm{~m}, \mathrm{~N} 43^{\circ} 13.94^{\prime} \mathrm{E} 131^{\circ} 59.95^{\prime} \text { (D. Gerus, A. Agafonov) }\end{array}$ \\
\hline 38 & $\begin{array}{l}\text { E. panormitanus; } \\
\text { H4152* }\end{array}$ & $\begin{array}{l}\text { StY } \\
\text { (Lu, Salomon, 1992) }\end{array}$ & USSR: Ukraine, Crimea \\
\hline 39 & $\begin{array}{l}\text { E. caucasicus; } \\
\mathrm{H} 3207^{*}\end{array}$ & $\begin{array}{l}\text { StY (Jensen, Wang, } \\
1991)\end{array}$ & USSR: Armenia, Dilidjan city outskirts \\
\hline 66 & $\begin{array}{l}\text { E. confusus; } \\
\text { BUM-0505 }\end{array}$ & $\begin{array}{l}\text { StH } \\
\text { (Lu et al., 1995) }\end{array}$ & $\begin{array}{l}\text { Russia, Republic of Buryatia, Tunka raion, Mondy village outskirts, the Tunka Range southern } \\
\text { slope, a stony brook bank, alt. } 1738 \mathrm{~m}, \mathrm{~N} 51^{\circ} 42.610^{\prime} \mathrm{E} 100^{\circ} 59.967^{\prime} \text { (D. Gerus, A. Agafonov) }\end{array}$ \\
\hline 67 & $\begin{array}{l}\text { E. confusus; } \\
\text { TAR- } 0730\end{array}$ & $»$ & $\begin{array}{l}\text { Russia, Republic of Tyva, Tes-Khem raion, Khorumnug-Taiga Mt. Range, Shuurmak-Samagaltay } \\
\text { Pass, spruce-larch forest, alt. } 1545 \mathrm{~m}, \mathrm{~N} 50^{\circ} 36.870^{\prime} \mathrm{E} 95^{\circ} 10.729^{\prime} \text { (I. Artemov) }\end{array}$ \\
\hline
\end{tabular}

Note. The numbering of accessions corresponds to the lane numbering in Fig. 2.

* Accessions kindly provided by Dr. B. Salomon (Swedish University of Agricultural Sciences, Department of Plant Breeding, Alnarp, Sweden). 
Table 2. Accessions of Elymus species with unknown genomic constitutions collected in Russia

\begin{tabular}{|c|c|c|}
\hline No. & $\begin{array}{l}\text { Elymus species; } \\
\text { accession }\end{array}$ & Location and collectors \\
\hline 18 & $\begin{array}{l}\text { E. uralensis; } \\
\text { UKU-1617 }\end{array}$ & $\begin{array}{l}\text { Republic of Bashkortostan, Beloretsk raion, Novoabzakovo village outskirts, dry meadow in a mixed forest, } \\
\text { alt. } 618 \mathrm{~m}, \mathrm{~N} 53^{\circ} 48.718^{\prime} \mathrm{E} 58^{\circ} 40.377^{\prime} \text { (A. Agafonov, S. Asbaganov) }\end{array}$ \\
\hline 19 & $\begin{array}{l}\text { E. viridiglumis; } \\
\text { UKU-1618 }\end{array}$ & $\begin{array}{l}\text { Republic of Bashkortostan, Beloretsk raion, Novoabzakovo village outskirts, tall herbage meadow in a birch } \\
\text { open stand, alt. } 619 \mathrm{~m}, \mathrm{~N} 53^{\circ} 48.718^{\prime} \mathrm{E} 58^{\circ} 40.377^{\prime} \text { (A. Agafonov, S. Asbaganov) }\end{array}$ \\
\hline 20 & $\begin{array}{l}\text { E. transbaicalensis; } \\
\text { AKU-0422 }\end{array}$ & $\begin{array}{l}\text { Altai Republic, Kosh-Agach raion, } 10 \mathrm{~km} \text { N of Chagan-Uzun village along the Chuya Highway, Kuyaktanar valley, } \\
\text { alt. } 1815 \mathrm{~m}, \mathrm{~N} 50^{\circ} 9.783^{\prime} \mathrm{E} 88^{\circ} 19.054^{\prime} \text { (A. Agafonov, D. Gerus) }\end{array}$ \\
\hline 21 & $\begin{array}{l}\text { E. transbaicalensis; } \\
\text { GAR-0530 }\end{array}$ & $\begin{array}{l}\text { Republic of Buryatia, Oka raion, the road to Orlik town, shingle bank of the Gargan River, } \\
\text { alt. } 1610 \mathrm{~m}, \mathrm{~N} 52^{\circ} 05.947^{\prime} \mathrm{E} 100^{\circ} 23.005^{\prime} \text { (A. Agafonov, D. Gerus) }\end{array}$ \\
\hline 22 & $\begin{array}{l}\text { E. margaritae; } \\
\text { GUK-1009 }\end{array}$ & $\begin{array}{l}\text { Altai Republic, Ust-Koksa raion, Krasnaya Mt., a complex of screes and highland meadows, } \\
\text { alt. } 2028 \mathrm{~m}, \mathrm{~N} 50^{\circ} 4.495^{\prime} \mathrm{E} 85^{\circ} 13.073^{\prime} \text { (D. Nikonova, E. Kobozeva) }\end{array}$ \\
\hline 23 & $\begin{array}{l}\text { E. margaritae; } \\
\text { AUK-0650 }\end{array}$ & $\begin{array}{l}\text { Altai Republic, Kosh-Agach raion, Ukok Plateau, stony meadow in a brook valley, } \\
\text { alt. } 2438 \mathrm{~m}, \mathrm{~N} 49^{\circ} 30.418^{\prime} \mathrm{E} 88^{\circ} 05.012^{\prime} \text { (A. Agafonov, B. Salomon) }\end{array}$ \\
\hline 24 & $\begin{array}{l}\text { E. komarovii; } \\
\text { AKU-0458 }\end{array}$ & $\begin{array}{l}\text { Altai Republic, Kosh-Agach raion, } 10 \mathrm{~km} \text { N of Chagan-Uzun village along the Chuya Highway, Kuyaktanar valley, } \\
\text { alt. } 1815 \mathrm{~m}, \mathrm{~N} 50^{\circ} 9.783^{\prime} \mathrm{E} 88^{\circ} 19.054^{\prime} \text { (A. Agafonov, D. Gerus) }\end{array}$ \\
\hline 25 & $\begin{array}{l}\text { E. transbaicalensis; } \\
\text { TUV-9697 }\end{array}$ & Republic of Tyva, Todzha raion, Azas State Nature Reserve, Ilgi-Chul ranger post (D. Shaulo) \\
\hline 28 & $\begin{array}{l}\text { E. komarovii; } \\
\text { AKT-0417 }\end{array}$ & $\begin{array}{l}\text { Altai Republic, Kosh-Agach raion, North-Chuya Range, Aktry Gorge, ground road edge at forest margin, } \\
\text { alt. } 2061 \mathrm{~m}, \mathrm{~N} 50^{\circ} 6.518^{\prime} \mathrm{E} 87^{\circ} 48.193^{\prime} \text { (A. Agafonov, D. Gerus) }\end{array}$ \\
\hline 29 & $\begin{array}{l}\text { E. komarovii; } \\
\text { GAR-0501 }\end{array}$ & $\begin{array}{l}\text { Republic of Buryatia, Oka raion, the Oka River valley, forest glade } 50 \mathrm{~m} \text { from the Gargan River mouth, } \\
\text { alt. } 1607 \mathrm{~m}, \mathrm{~N} 52^{\circ} 05.947^{\prime} \mathrm{E} 100^{\circ} 23.005^{\prime} \text { (A. Agafonov, D. Gerus) }\end{array}$ \\
\hline 30 & $\begin{array}{l}\text { E. komarovii; } \\
\text { JPO-1505 }\end{array}$ & $\begin{array}{l}\text { Republic of Sakha-Yakutia, Khangalas raion, Pokrovsk Town outskirts, a meadow at the gas station, } \\
\text { alt. } 131 \mathrm{~m}, \mathrm{~N} 61^{\circ} 29.367^{\prime} \mathrm{E} 129^{\circ} 08.225^{\prime} \text { (E. Kobozeva, S. Asbaganov) }\end{array}$ \\
\hline 31 & $\begin{array}{l}\text { E. subfibrosus; } \\
\text { ANA-1118 }\end{array}$ & Chukotskiy Autonomous district, Anadyr'Town outskirts (D. Lysenko) \\
\hline 32 & $\begin{array}{l}\text { E. subfibrosus; } \\
\text { LEN-1524 }\end{array}$ & $\begin{array}{l}\text { Republic of Sakha-Yakutia, Khangalas raion, the nature reserve "Lenskie Stolby", } \\
\text { alt. } 156 \mathrm{~m}, \mathrm{~N} 61^{\circ} 6.370^{\prime} \mathrm{E} 127^{\circ} 21.593^{\prime} \text { (E. Kobozeva, S. Asbaganov) }\end{array}$ \\
\hline 33 & $\begin{array}{l}\text { E. macrourus; } \\
12-0135\end{array}$ & $\begin{array}{l}\text { Taymyr Peninsula, the shingle floodplain of the Bolshaya Lesnaya Rassokha River at its mouth, } \\
\text { alt. } 2 \mathrm{~m}, \mathrm{~N} 72^{\circ} 37.363^{\prime} \mathrm{E} 101^{\circ} 17.793^{\prime} \text { (E. Pospelova) }\end{array}$ \\
\hline 34 & $\begin{array}{l}\text { E. jacutensis; } \\
13-0443\end{array}$ & $\begin{array}{l}\text { Taymyr Peninsula, the Anabar Plateau margin, Eriechka and Nyamakit-Daldyn Rivers, a small meadow below } \\
\text { rocks, alt. } 218 \mathrm{~m}, \mathrm{~N} 71^{\circ} 15.250^{\prime} \mathrm{E} 105^{\circ} 37.452^{\prime} \text { (I. Pospelov) }\end{array}$ \\
\hline 35 & $\begin{array}{l}\text { E. sajanensis; } \\
\text { ZUN-0502 }\end{array}$ & $\begin{array}{l}\text { Republic of Buryatia, Oka raion, Zun-Kholbo village outskirts, } \\
\text { alt. } 1682 \mathrm{~m}, \mathrm{~N} 52^{\circ} 10.092^{\prime} \mathrm{E} 100^{\circ} 57.581^{\prime} \text { (A. Agafonov, D. Gerus) }\end{array}$ \\
\hline 36 & $\begin{array}{l}\text { E. sajanensis; } \\
\text { ART-0202 }\end{array}$ & $\begin{array}{l}\text { Altai Republic, Kosh-Agach raion, Chikhacheva Range, shingle bank of a Buguzun River left tributary, } \\
\text { alt. } 2254 \mathrm{~m}, \mathrm{~N} 50^{\circ} 1.914^{\prime} \mathrm{E} 89^{\circ} 23.620^{\prime} \text { (I. Artemov) }\end{array}$ \\
\hline 37 & $\begin{array}{l}\text { E. amurensis; } \\
\text { MES-1111 }\end{array}$ & $\begin{array}{l}\text { Primorskiy Krai, Khasan raion, Andreevka village outskirts, meadow patch at a ground road edge, } \\
\text { alt. } 93 \mathrm{~m}, \mathrm{~N} 42^{\circ} 37.045^{\prime} \mathrm{E} 131^{\circ} 8.650^{\prime} \text { (E. Kobozeva, A. Agafonov) }\end{array}$ \\
\hline 40 & $\begin{array}{l}\text { E. transbaicalensis; } \\
\text { AKT-0628 }\end{array}$ & $\begin{array}{l}\text { Altai Republic, Kosh-Agach raion, North-Chuya Range, Aktry Gorge, willow thickets at the mountaineering camp, } \\
\text { alt. } 2118 \mathrm{~m}, \mathrm{~N} 50^{\circ} 5.038^{\prime} \mathrm{E} 87^{\circ} 46.820^{\prime} \text { (A. Agafonov, D. Gerus) }\end{array}$ \\
\hline 41 & $\begin{array}{l}\text { E. kronokensis; } \\
\text { BER-0804 }\end{array}$ & $\begin{array}{l}\text { Republic of Buryatia, Eravnoe raion, SE of the temporary settlement Ozernyy, larch forest, } \\
\text { alt. } 1154 \mathrm{~m}, \mathrm{~N} 52^{\circ} 58.625^{\prime} \mathrm{E} 111^{\circ} 38.166^{\prime} \text { (O. Anenkhonov) }\end{array}$ \\
\hline 42 & $\begin{array}{l}\text { E. kronokensis; } \\
\text { MMA-1103 }\end{array}$ & $\begin{array}{l}\text { Magadan oblast, Madaun village outskirts, a burnt area in the Arman' Rover floodplain, } \\
\text { alt. } 627 \mathrm{~m}, \mathrm{~N} 60^{\circ} 35.861^{\prime} \mathrm{E} 150^{\circ} 40.862^{\prime} \text { (D. Lysenko) }\end{array}$ \\
\hline 43 & $\begin{array}{l}\text { E. kronokensis; } \\
\text { KES-9603 }\end{array}$ & $\begin{array}{l}\text { Kamchatka Krai, Bystraya raion, southern slope of a mountain } N \text { of Esso village, } \\
\text { alt. } 627 \mathrm{~m}, \mathrm{~N} 55^{\circ} 55.945^{\prime} \mathrm{E} 158^{\circ} 41.275^{\prime} \text { (A. Agafonov, B. Salomon) }\end{array}$ \\
\hline 44 & $\begin{array}{l}\text { E. lenensis; } \\
12-0125\end{array}$ & $\begin{array}{l}\text { Taymyr Peninsula, Bolshaya Rassokha and Novaya Rivers, } \\
\text { alt. } 39 \mathrm{~m}, \mathrm{~N} 72^{\circ} 39.613^{\prime} \mathrm{E} 101^{\circ} 17.079^{\prime} \text { (I. Pospelov) }\end{array}$ \\
\hline 45 & $\begin{array}{l}\text { E. kamczadalorum; } \\
\text { KSO-9623 }\end{array}$ & $\begin{array}{l}\text { Kamchatka Krai, Elizovo raion, Sosnovka village outskirts, } \\
\text { alt. } 247 \mathrm{~m}, \mathrm{~N} 53^{\circ} 5.046^{\prime} \mathrm{E} 158^{\circ} 17.918^{\prime} \text { (A. Agafonov, B. Salomon) }\end{array}$ \\
\hline 46 & $\begin{array}{l}\text { E. charkeviczii; } \\
\text { KES- } 9670\end{array}$ & $\begin{array}{l}\text { Kamchatka Krai, Bystraya raion, Esso village outskirts, ground road margin, } \\
\text { alt. } 484 \mathrm{~m}, \mathrm{~N} 55^{\circ} 55.014^{\prime} \mathrm{E} 158^{\circ} 42.116^{\prime} \text { (A. Agafonov, B. Salomon) }\end{array}$ \\
\hline 47 & $\begin{array}{l}\text { E. charkeviczii; } \\
\text { MSN-1202 }\end{array}$ & $\begin{array}{l}\text { Magadan City, Snezhnyy settlement, path at a forest margin, } \\
\text { alt. } 145 \mathrm{~m}, \mathrm{~N} 59^{\circ} 43.466^{\prime} \mathrm{E} 150^{\circ} 52.677^{\prime} \text { (N. Badmaeva) }\end{array}$ \\
\hline 52 & $\begin{array}{l}\text { E. lenensis; } \\
\text { LEN-1520 }\end{array}$ & $\begin{array}{l}\text { Republic of Sakha (Yakutia), Khangalas raion, meadow slope at the Lena River right bank, } \\
\text { alt. } 114 \mathrm{~m}, \mathrm{~N} 61^{\circ} 6.369^{\prime} \mathrm{E} 127^{\circ} 21.593^{\prime} \text { (E. Kobozeva, S. Asbaganov) }\end{array}$ \\
\hline
\end{tabular}


Table 2 (end)

\begin{tabular}{|c|c|c|}
\hline No. & $\begin{array}{l}\text { Elymus species; } \\
\text { accession }\end{array}$ & Location and collectors \\
\hline 53 & $\begin{array}{l}\text { E. lenensis; } \\
\text { ALD-1539-3 }\end{array}$ & $\begin{array}{l}\text { Republic of Sakha (Yakutia), Aldan raion, the Aldan River bank, shrubbery at a sandy bank, } \\
\text { alt. } 228 \mathrm{~m}, \mathrm{~N} 58^{\circ} 40.878^{\prime} \mathrm{E} 128^{\circ} 33.081^{\prime}(\mathrm{E} . \mathrm{K} \text { Kobozeva, S. Asbaganov) }\end{array}$ \\
\hline 54 & $\begin{array}{l}\text { E. kronokensis; } \\
\text { KRT-1611 }\end{array}$ & $\begin{array}{l}\text { Krasnoyarsk Kray, Evenk raion, Tura village outskirts, the Nizhnyaya Tunguska River, path side in a larch forest, } \\
\text { alt. } 169 \mathrm{~m}, \mathrm{~N} 64^{\circ} 16.478^{\prime} \mathrm{E} 100^{\circ} 16.445^{\prime}(\mathrm{L} \text {. Krivobokov) }\end{array}$ \\
\hline 55 & $\begin{array}{l}\text { E. kronokensis; } \\
12-0137\end{array}$ & $\begin{array}{l}\text { Taymyr Peninsula, the Bolshaya Rassokha River bank bluff, alt. } 2 \text { m, N 72³5.808' E } 101^{\circ} 15.900^{\prime} \\
\text { (E. Pospelova) }\end{array}$ \\
\hline 56 & $\begin{array}{l}\text { E. kronokensis; } \\
\text { TAL-0602 }\end{array}$ & $\begin{array}{l}\text { Altai Republic, Kosh-Agach raion, the Taldura River valley, larch forest on a mountain slope, } \\
\text { alt. } 2095 \mathrm{~m}, \mathrm{~N} 49^{\circ} 57.472^{\prime} \mathrm{E} 87^{\circ} 57.552^{\prime} \text { (D. Gerus, A. Agafonov) }\end{array}$ \\
\hline 57 & $\begin{array}{l}\text { E. subfibrosus; } \\
\text { KRT-1612 }\end{array}$ & $\begin{array}{l}\text { Krasnoyarsk Kray, Evenk raion, Tura village, a ground road edge, } \\
\text { alt. } 309 \mathrm{~m}, \mathrm{~N} 64^{\circ} 16.920^{\prime} \mathrm{E} 100^{\circ} 14.880^{\prime}(\mathrm{L} . \text { Krivobokov) }\end{array}$ \\
\hline 58 & $\begin{array}{l}\text { E. subfibrosus; } \\
\text { JRO-1733 }\end{array}$ & $\begin{array}{l}\text { Republic of Sakha (Yakutia), Tompo raion, the Kolyma Riad, Verkhoyanskiy Mt. Range southern spurs, } \\
\text { the Rosomakha River valley, alt. } 460 \mathrm{~m}, \mathrm{~N} 63^{\circ} 2.879^{\prime} \mathrm{E} 137^{\circ} 52.610^{\prime} \text { (N. Badmaeva) }\end{array}$ \\
\hline 59 & $\begin{array}{l}\text { E. jacutensis; } \\
\text { ALU-1711 }\end{array}$ & $\begin{array}{l}\text { Altai Republic, Ulagan raion, herbaceous meadow at the Chulyshman River left bank under Katu-Yaryk Pass, } \\
\text { alt. } 733 \mathrm{~m}, \mathrm{~N} 50^{\circ} 55.497^{\prime} \mathrm{E} 088^{\circ} 12.226^{\prime}(\mathrm{E} . \text { Kobozeva) }\end{array}$ \\
\hline 60 & $\begin{array}{l}\text { E. jacutensis; } \\
\text { GAN-1516 }\end{array}$ & $\begin{array}{l}\text { Altai Republic, Chemal raion, Anos village outskirts, slope above the Anos River left bank at the bridge, } \\
\text { alt. } 380 \mathrm{~m}, \mathrm{~N} 51^{\circ} 30.014^{\prime} \mathrm{E} 85^{\circ} 57.160^{\prime} \text { (E. Kobozeva) }\end{array}$ \\
\hline 61 & $\begin{array}{l}\text { E. jacutensis; } \\
\text { ALD-1541 }\end{array}$ & $\begin{array}{l}\text { Republic of Sakha (Yakutia), Aldan raion, the Aldan River bank, shrubbery at a sandy bank, } \\
\text { alt. } 228 \mathrm{~m}, \mathrm{~N} 58^{\circ} 40.878^{\prime} \mathrm{E} 128^{\circ} 33.081^{\prime}(\mathrm{E} . \mathrm{K} \text { Kobozeva, S. Asbaganov) }\end{array}$ \\
\hline 62 & $\begin{array}{l}\text { E. macrourus; } \\
\text { MTE-1210 }\end{array}$ & $\begin{array}{l}\text { Magadan oblast, Tenka raion, roadside at a mixed forest margin, } \\
\text { alt. } 970 \mathrm{~m}, \mathrm{~N} 60^{\circ} 26.034^{\prime} \mathrm{E} 150^{\circ} 58.558^{\prime}(\mathrm{N} . \text { Badmaeva) }\end{array}$ \\
\hline 63 & $\begin{array}{l}\text { E. macrourus; } \\
\text { LEN-1524_1 }\end{array}$ & $\begin{array}{l}\text { Republic of Sakha (Yakutia), Khangalas raion, meadow slope at the Lena River right bank, } \\
\text { alt. } 114 \mathrm{~m}, \mathrm{~N} 61^{\circ} 6.369^{\prime} \mathrm{E} 127^{\circ} 21.593^{\prime} \text { (E. Kobozeva, S. Asbaganov) }\end{array}$ \\
\hline 64 & $\begin{array}{l}\text { E. turuchanensis; } \\
\text { KRE-1440 }\end{array}$ & $\begin{array}{l}\text { Krasnoyarsk Kray, Turukhansk raion, Bor village, Yenisey River sandy bank, } \\
\text { alt. } 30 \mathrm{~m}, \mathrm{~N} 61^{\circ} 36.265^{\prime} \mathrm{E} 90^{\circ} 0.143^{\prime}(\mathrm{M} \text {. Lomonosova) }\end{array}$ \\
\hline 65 & $\begin{array}{l}\text { E. peschkovae; } \\
\text { MJA-1106 }\end{array}$ & $\begin{array}{l}\text { Magadan oblast, Khasyn raion, Yablonovyy Pass, floodplain meadow at a road, } \\
\text { alt. } 755 \mathrm{~m}, \mathrm{~N} 60^{\circ} 19.467^{\prime} \mathrm{E} 151^{\circ} 10.540^{\prime} \text { (D. Lysenko) }\end{array}$ \\
\hline
\end{tabular}

Note. The numbering of accessions corresponds to the lane numbering in Fig. 2.

$1.7 \%$ agarose gel in TAE buffer. Molecular weight marker: $100+$ bp DNA Ladder (Evrogen, RF).

\section{Results and discussion}

The comparative analysis of sequences of the $\beta$ amylase gene published in R. Mason-Gamer (2013) showed that the studied fragment of $\mathrm{Y}$ genome of about $1100 \mathrm{bp}$ in length did not contain recognition sites for TaqI endonuclease, while St genome contained one recognition site in the fragment of interest at a distance of about $170 \mathrm{bp}$ from the primer El_balg_R4. The same site was present in some $\mathrm{H}$ genomes; besides, all $\mathrm{H}$ genomes contained a recognition site at a distance of about $280 \mathrm{bp}$ from the primer El_balg_F4. Visualized on gels, restriction patterns of the studied genomes were differentiated according to the lengths of the longest fragments: $\mathrm{H}$ genome was distinguished by the presence of a band at about $650 \mathrm{bp}$; St genome, 930 bp; and Y genome, 1100 bp (Fig. 1).

Restriction patterns of the CAPS marker employed were studied in 68 accessions (see Tables 1,2). Electrophoretic patterns formed after TaqI digestion are shown in Fig. 2. Based on the results of CAPS analysis, genomic constitutions of the accessions studied were determined. Previously known genomic constitutions were confirmed in 15 species of $16, E$. $k a-$ moji being the only exception. In 16 species, genomic compositions were determined for the first time: 15 of them had the

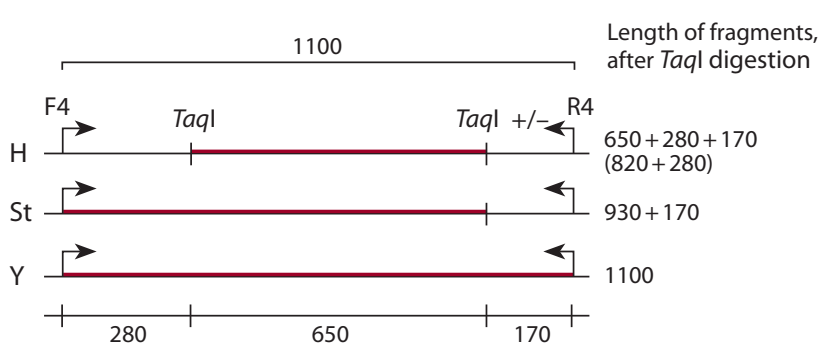

Fig. 1. Map of recognition sites for Taql endonuclease in the $\beta$ amylase gene fragment amplified from the basic haplomes constituting the polyploid Elymus genome.

genomic constitution StStHH, and one species, E. amurensis, had StStYY (Table 3). However, some limitations of the approach were met. For example, in two accessions of E. kamoji CAPS-analysis revealed only two haplomes, St and Y (Fig. 2, lanes 1 and 2), whereas it is known to be hexaploid according to the number of chromosomes, thus, it should contain three basic genomes (haplomes). It is improbable that the absence of restriction fragments corresponding to haplome $\mathrm{H}$ was due to incomplete digestion. Since all representatives of the genus contain St haplome, possessing a recognition site for TaqI endonuclease, the presence of St-specific fragments serves as an 

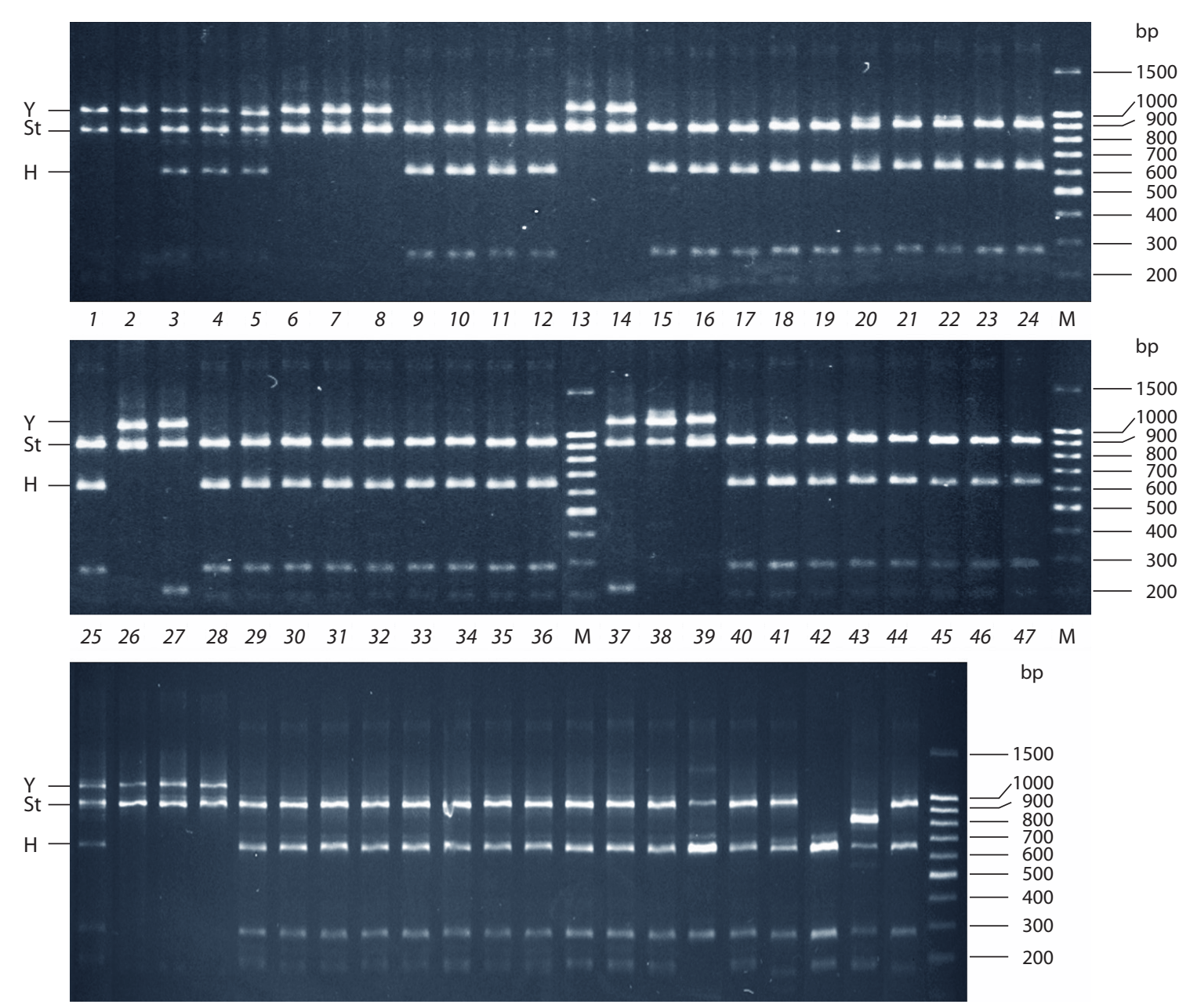

$\begin{array}{llllllllllllllllllllll}48 & 49 & 50 & 51 & 52 & 53 & 54 & 55 & 56 & 57 & 58 & 59 & 60 & 61 & 62 & 63 & 64 & 65 & 66 & 67 & 68 & M\end{array}$

Fig. 2. Polymorphism of restriction fragment lengths (CAPS) after Taql digestion of the PCR-amplified fragment of the $\beta$ amylase gene in species of the genus Elymus.

Lane numbers correspond to the accession numbering in Tables 1 and 2. $M$ - molecular weight ladder: 100+bp DNA Ladder (Evrogen).

Table 3. The list of boreal Elymus species in Asian Russia in which genome constitutions (GC) were determined by the CAPS method

\begin{tabular}{|c|c|c|c|c|c|c|c|}
\hline No. & Elymus species & $\begin{array}{l}\text { Number } \\
\text { of accessions studied }\end{array}$ & GC & No. & Elymus species & $\begin{array}{l}\text { Number } \\
\text { of accessions studied }\end{array}$ & GC \\
\hline 1 & E. amurensis & 1 & StY & 9 & E. margaritae & 2 & StH \\
\hline 2 & E. charkeviczii & 2 & $\mathrm{StH}$ & 10 & E. sajanensis & 2 & StH \\
\hline 3 & E. jacutensis & 4 & StH & 11 & E. subfibrosus & 4 & StH \\
\hline 4 & E. kamczadalorum & 1 & $\mathrm{StH}$ & 12 & E. transbaicalensis & 4 & StH \\
\hline 5 & E. komarovii & 4 & $\mathrm{StH}$ & 13 & E. uralensis & 1 & StH \\
\hline 6 & E. kronokensis & 6 & $\mathrm{StH}$ & 14 & E. viridiglumis & 1 & StH \\
\hline 7 & E. lenensis & 3 & $\mathrm{StH}$ & 15 & E. turuchanensis & 1 & StH \\
\hline 8 & E. macrourus & 3 & $\mathrm{StH}$ & 16 & E. peschkovae & 2 & StH \\
\hline
\end{tabular}

internal control for the completeness of hydrolysis. According to the classification system based on genomic compositions, E. kamoji belongs to the genus Campeiostachys (Baum et al., 2011) which embraces species with the genomic composition StHY. In fact, we performed a cytological analysis, which showed that both accessions of E. kamoji possessed the chromosome number $2 n=42$, corresponding to hexaploid. The presence of the $\mathrm{H}$ genome lacking two recognition sites for TaqI endonuclease in E. kamoji brings its origin into a question. It is not inconceivable that different representatives of 
the genus received their $\mathrm{H}$ genomes from different ancestor species, which agrees with the assumption of polyphyly of the donors of basic haplomes (Mason-Gamer, 2013).

An interesting pattern of restriction fragments was observed in two accessions of E. confusus (see Fig. 2, lanes 66 and 67 ), with the genome constitution formerly determined as StStHH (Lu et al., 1995). In accession TAR-0730 (see Fig. 2, lane 67), the longer fragment corresponding to the allele from St genome is truncated, possibly, as the result of a deletion or acquisition of an additional restriction site. The spectrum of restriction fragments in accession BUM-0505 (see Fig. 2, lane 66) lacks the fragment of about $930 \mathrm{bp}$ characteristic of St genome, while the smaller fragment of about $170 \mathrm{bp}$ corresponding to this haplome is clearly seen. This phenomenon might be attributed to a mutation in the St genome of the accession, for example, appearance of a recognition site for TaqI. Another possibility is a recombination and/or introgression between genomes St and $\mathrm{H}$ in the course of intense microevolutionary processes indirectly confirmed by the high morphologic variability within this species.

According to the CAPS analysis undertaken in the present work, almost all newly studied accessions of the boreal group of species from Siberia and Russian Far East have the $\mathrm{StH}$ genomic composition. One exception was E. amurensis, phylogenetically close to the StY-genomic species E. ciliaris and possessing the genome composition StY. This implies that the center of species diversity of the Asiatic StH-genome group is shifted to the north as compared to that of the StYgenome group, which is considered to be situated in China (Lu, Salomon, 1992). In this context, it is worth noting that in North America, the genus Elymus is also represented mainly by StH-genome species (except for Elymus californicus with unclear origin) (Mason-Gamer, 2001). Besides, in that territory a number of adventive Asiatic StHY- and StY-genome species were found (Barkworth et al., 2007).

In general, the applied method showed a high accuracy: in the present work earlier known genome constitutions were confirmed by CAPS analysis in 15 Elymus species of 16 . For 10 species, the genomic composition newly determined by CAPS analysis as $\mathrm{StH}$, was independently corroborated by sequencing of a cloned fragment of the GBSS1 (waxy) gene (Kobozeva et al., 2018; Agafonov et al., 2019). It should be noted that the sequencing of DNA from polyploid species has a disadvantage, as it is rather laborious, requiring additional gene cloning manipulations.

\section{Conclusion}

The main advantage of CAPS markers is the ease of their methodic implementation, which permits one to analyze many specimens with extensive morphologic and genetic variability from broad ranges. The present work involves CAPS analysis with the use of a fragment of the gene for $\beta$ amylase and demonstrates rather good predictive power of the method. However, it should be kept in mind that no molecular marker taken by itself can unambiguously identify a genome or species; it serves as a marker, not diagnostic. Therefore, the development of additional simple and accessible approaches for genome identification in new and poorly studied biotypes from local habitats remains vital.

\section{References}

Agafonov A.V., Asbaganov S.V., Shabanova (Kobozeva) E.V., Morozov I.V., Bondar A.A. Genome constitution and differentiation of subgenomes in Siberian and Far Eastern endemic species of the genus Elymus (Poaceae) according to the sequencing of the nuclear gene waxy. Vavilovskii Zhurnal Genetiki i Selektsii = Vavilov Journal of Genetics and Breeding. 2019;23(7):817-826. DOI 10.18699/ VJ19.555. (in Russian)

Agafonov A.V., Kobozeva E.V., Asbaganov S.V., Shmakov N.A. Current achievements and prospects of construction of a phylogenetically oriented taxonomy of the genus Elymus (Poaceae: Triticeae). Proceedings of the 14th International Scientific and Practical Conference "Problems of Botany of Southern Siberia and Mongolia". Barnaul. 2015;314-322. (in Russian)

Barkworth M.E. Changing perceptions of the Triticeae. In: Jacobs S.W.L., Everett J. (Eds.). Grasses: Systematics and Evolution. Melbourne: CSIRO, 2000;110-120.

Barkworth M.E., Cambell J.J.N., Salomon B. Elymus L. In: Barkworth M.E., Capels K.M., Long S., Anderton L.K., Piep M.B. (Eds.). Flora of North America. New York; Oxford: Oxford University Press, 2007;24:288-343.

Baum B.R., Yang J.-L., Yen C., Agafonov A.V. A taxonomic revision of the genus Campeiostachys Drobov. J. Syst. Evol. 2011;49(2):146159.

Dewey D.R. Synthetic Agropyron-Elymus hybrids. III. Elymus canadensis $\times$ Agropyron caninum, A. trachycaulum and A. striatum. Amer. J. Bot. 1968;55:1133-1139.

Dewey D.R. Cytogenetics of Elymus sibiricus and its hybrids with Agropyron tauri, Elymus canadensis and Agropyron caninum. Bot. Gaz. 1974;135:80-87.

Dewey D.R. Cytogenetics of Agropyron ugamicum and six of its interspecific hybrids. Bot. Gaz. 1980;141:305-312.

Dewey D.R. The genomic system of classification as a guide to intergeneric hybridization with the perennial Triticeae. In: Gustafson J.P. (Ed.). Gene Manipulation in Plant Improvement. New York: Plenum Publ. Corp., 1984;209-279.

Gostimsky S.A., Kokaeva Z.G., Konovalov F.A. Studying plant genome variation using molecular markers. Russ. J. Genet. 2005;41(4): 378-388. DOI 10.1007/s11177-005-0101-1.

Hu C.Y., Tsai Y.Z., Lin S.F. Development of STS and CAPS markers for variety identification and genetic diversity analysis of tea germplasm in Taiwan. Bot. Stud. 2014;55:12. DOI 10.1186/1999-3110-55-12.

Jensen K.B. Cytology, fertility, and origin of Elymus abolinii (Drob.) Tzvelev and its $\mathrm{F}_{1}$ hybrids with Pseudoroegneria spicata, E. lanceolatus, E. dentatus ssp. ugamicus, and E. drobovii (Poaceae: Triticeae). Genome. 1989;32:468-474. DOI 10.1139/g89-470.

Jensen K.B. Cytology and morphology of Elymus pendulinus, E. pendulinus ssp. multiculmis, and E. parviglume (Poaceae: Triticeae). Bot. Gaz. 1990;151(2):245-251.

Jensen K.B., Hatch S. Genome analysis, morphology, and taxonomy of Elymus gmelinii and E. strictus (Poaceae: Triticeae). Bot. Gaz. 1989;150(1):84-92.

Jensen K.B., Wang R.R.C. Cytogenetics of Elymus caucasicus and Elymus longearistatus (Poaceae: Triticeae). Genome. 1991;34:860867.

Kobozeva E.V., Asbaganov S.V., Agafonov A.V. Genome composition and assessment of the divergence between Russian boreal species in the genus Elymus (Poaceae) detected on the basis of sequencing of the nuclear gene GBSSI. In: Prospects of Development and Challenges of Modern Botany. BIO Web Conf. 2018;11.00023. DOI 10.1051/bioconf/20181100023.

Kobozeva E.V., Mglinets A.V., Agafonov A.V. Identification of the genomic composition in allopolyploid species of the genus Elymus (Poaceae: Triticeae) with CAPS-analysis. In: Proceedings of the 6th International Conference "Issues in the Study of the Vegetation Cover in Siberia”. Tomsk. 2017;155-157. DOI 10.17223/ 9785946216371/51. (in Russian) 
Konieczny A., Ausubel F.M. A procedure for mapping Arabidopsis mutations using co-dominant ecotype-specific PCR-based markers. Plant J. 1993;4(2):403-410.

Li X.-M., Lee B.S., Mammadov A.C., Koo B.-C., Mott I.W., Wang R.R.C. CAPS markers specific to Eb, Ee, and $\mathrm{R}$ genomes in the tribe Triticeae. Genome. 2007;50:400-411.

Liu C.W., Dewey D.R. The genome constitution of Elymus fedtschenkoi. Acta Genet. Sinica. 1983;10:20-27.

Löve A. Conspectus of the Triticeae. Feddes Repert. 1984;95:425-521.

Lu B.-R., Bothmer R. von. Interspecific hybridization with Elymus himalayanus and E. schrenkianus, and other Elymus species (Triticeae: Poaceae). Genome. 1992;35:230-237.

Lu B.-R., Salomon B. Differentiation of the SY genomes in Asiatic Elymus. Hereditas. 1992;116:121-126.

Lu B.-R., Salomon B., Bothmer R. von. Interspecific hybridization with Elymus confusus and E. dolichaterus, and their genomic relationships (Poaceae: Triticeae). Plant Syst. Evol. 1995;197:1-17.

Mason-Gamer R.J. Origin of North American Elymus (Poaceae: Triticeae) allotetraploids based on granule-bound starch synthase gene sequences. Syst. Bot. 2001;26:757-768.
Mason-Gamer R.J. Phylogeny of a genomically diverse group of Elymus (Poaceae) allopolyploids reveals multiple levels of reticulation. PLoS One. 2013;8(11):e78449. DOI 10.1371/journal.pone.0078449.

Okito P., Mott I.W., Wu Y., Wang R.R. A Y genome specific STS marker in Pseudoroegneria and Elymus species (Triticeae: Gramineae). Genome. 2009;52(4):391-400.

Shavrukov Y.N. CAPS markers in plant biology. Russ. J. Genet.: Appl. Res. 2016;6(3):279-287. DOI 10.1134/S2079059716030114.

Tsvelyov N.N. On the genus Elymus (Poaceae) in Russia. Botanicheskiy Zhurnal $=$ Botanical Journal. 2008;93(10):1587-1596. (in Russian)

Tsvelyov N.N., Probatova N.S. The genera Elymus L., Elytrigia Desv., Agropyron Gaertn., Psathyrostachys Nevski, and Leymus Hochst. (Poaceae: Triticeae) in the flora of Russia. In: V.L. Komarov Memorial Lectures. Vladivostok: Dalnauka Publ., 2010;57:5-102. (in Russian)

Zhou Y.-H., Wu B.-H., Fu T.-H., Zheng Y.-L. Morphology, fertility and cytogenetics of intergeneric hybrid between Roegneria kamoji Ohwi and Dasypyrum villosum (L.) Candargy (Poaceae: Triticeae). J. Syst. Evol. 1999;37(2):125-130.

ORCID ID

A.V. Agafonov orcid.org/0000-0002-1403-5867

S.V. Asbaganov orcid.org/0000-0002-7482-7495

Acknowledgements. This work was supported by the state project "Estimation of the morphogenetic potential of the North Asian plant population by experimental methods" (state registration number: AAAA-A17-117012610051-5) for the Central Siberian Botanical Garden (CSBG) SB RAS and state project 0324-2019-0039-C-01 for the Institute of Cytology and Genetics SB RAS, the Russian Foundation for Basic Research (project No. 18-04-01030). Materials of the bioresource scientific collection of the CSBG SB RAS "Collections of living plants in open and closed ground", USU No. 440534 were used.

Conflict of interest. The authors declare no conflict of interest.

Received December 25, 2018. Revised August 8, 2019. Accepted October 30, 2019. 